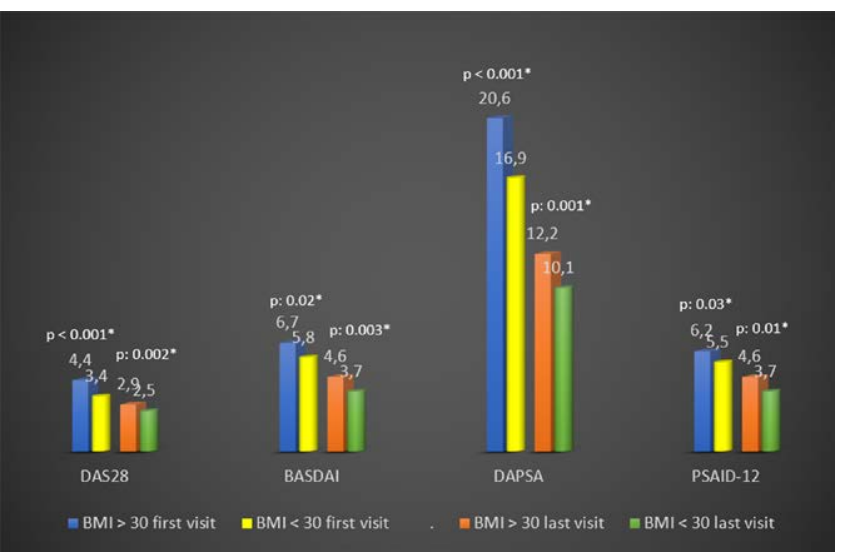

Figure 1. Baseline and last visit disease activity by BMl categoriesp: $0.03^{*} \mathrm{p}: 0.01^{*} \mathrm{p}: 0.001^{*} \mathrm{p}$ : $0.002^{* *} p<0.05 B M I$ : Body mass Index, DAS: Disease Activity Score, BASDAI: Bath Ankylosing Spondylitis Disease Activity Index, DAPSA: Disease Activity index for Psoriatic Arthritis, PSAID: Psoriatic Arthritis Impact of Disease

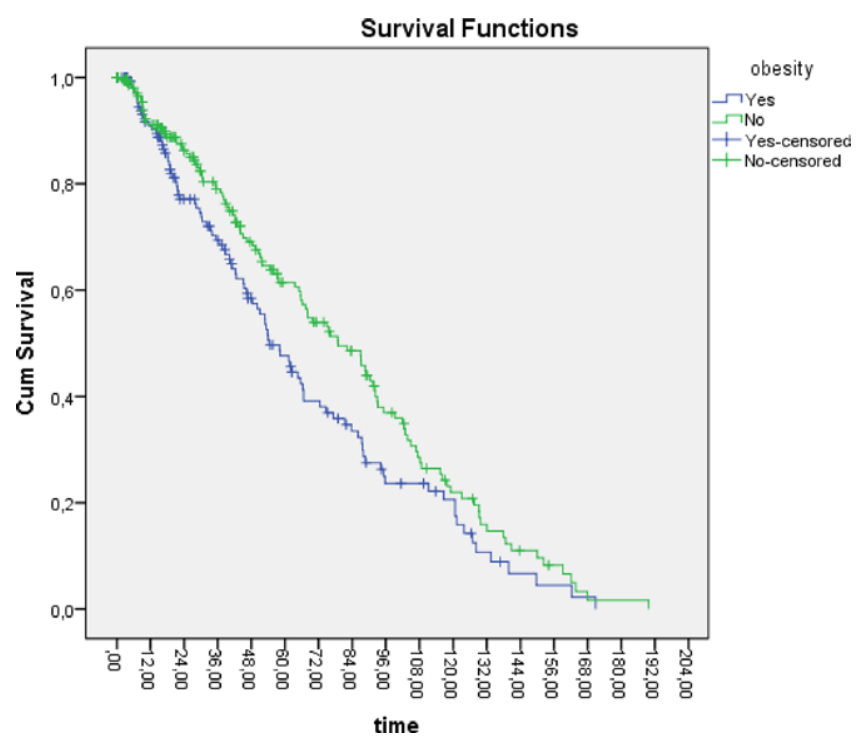

Figure 2. Retention rate for biological DMARD by BMI categoriesMedian of retention rate of first bDMARD: BMI $>30 \mathrm{~kg} / \mathrm{m}^{2}$ and $<30 \mathrm{~kg} / \mathrm{m}^{2} 54.2$ and 79 , respectively.Log rank p-value between $\mathrm{BMl}>30 \mathrm{~kg} / \mathrm{m}^{2}$ and $<30 \mathrm{~kg} / \mathrm{m}^{2}: 0.03^{*}$

Disclosure of Interests: Bayram Farisoğulları: None declared, Gözde Kübra Yardımcı: None declared, Ertugrul Cagri Bolek: None declared, Emre Bilgin: None declared, Emine Duran: None declared, Gizem Ayan: None declared, Levent Kılıç: None declared, Omer Karadag: None declared, Ali Akdoğan: None declared, Şule Apraş Bilgen: None declared, Ali insan Ertenli: None declared, Sedat Kiraz: None declared, Umut Kalyoncu Consultant of: Abbvie, Amgen, Janssen, Lilly, Novartis, UCB

DOI: 10.1136/annrheumdis-2020-eular.4375

\section{AB0764 $\quad$ SAFETY OF SYSTEMIC CORTICOSTEROIDS IN A SHORT REGIMEN IN PATIENTS WITH PSORIATIC ARTHRITIS. RETROSPECTIVE ANALYSIS OF A LARGE OBSERVATIONAL COHORT.}

L. Farran Ortega ${ }^{1}$, M. Tormo Ratera ${ }^{1}$, J. Lluch Pons ${ }^{1}$, M. Mora ${ }^{1}$, C. Marco Pascual $^{1}$, X. González Giménez ${ }^{1}$, J. M. Nolla ${ }^{1}$, J. Rodríguez ${ }^{1}{ }^{1}$ Hospital Universitari de Bellvitge, Rheumatology, Hospitalet de Llobregat, Spain

Background: There is controversy surrounding the use of systemic corticosteroids in psoriatic arthritis (PsA). It's an accepted fact that the use of systemic corticosteroids can trigger severe flare-up of erythroderma or pustular psoriasis. Nevertheless, corticosteroids have been used to achieve faster improvement of joint symptoms of PsA.

Objectives: To analyze the use of systemic corticosteroids at intermediate doses in a short regimen in patients with PsA, as well as the serious complications of psoriasis upon withdrawal.
Methods: Retrospective analysis of an observational cohort of 453 patients from a university hospital, following a specific protocol from 1992 to 2019. The following variables have been collected: corticosteroid treatment (methylprednisolone $\leq 16 \mathrm{mg}$ /day in a slow tapering regimen in 2 months), demographic and disease factors, comorbidities that could be associated (diabetes mellitus, high blood pressure, severe infections) and serious complications of psoriasis (erythroderma or pustular psoriasis). To assess the activity of psoriasis, physician global assessment is mostly used and occasionally to a lesser extent body surface area and psoriasis area severity index.

Statistical analysis (SPSS v.25): descriptive analysis, Chi-squared test for qualitative variables and t-student test for quantitative variables.

Results: In our series, 35.98\% (163/453) of patients have received short cor ticosteroid regimen at some point in follow-up care, of which $93.8 \%$ received concomitant treatment with disease modifying antirheumatic drugs (DMARD).

Only $6.2 \%$ of the patients who received short corticosteroid regimen presented a flare-up of psoriasis, most of them mildly. No patient developed an erythroderma or severe pustular psoriasis.

After analyzing the data, a greater use of this regimen of treatment has been observed in patients with dactylitis $(44.6 \%$ with dactylitis vs $27.8 \%$ without dactylitis, $p<0.001)$ and a lower use of corticosteroids in axial PsA ( $14 \%$ of axial PsA vs $41 \%$ of non-axial PsA, $p<0.001$ ).

There were no significant differences in the use of corticosteroids in respect to sex, age, age of onset of PsA, duration of PsA or high blood pressure. Nor in factors of poor radiographic prognosis: number of damaged joints, mutilating PsA and carpitis.

Conclusion: In our series, no patient developed an erythroderma or severe pustular psoriasis and most of the flare-ups of psoriasis were mild. The use of systemic corticosteroids at intermediate doses in a slow tapering regimen concomitantly with DMARD can be safely used in patients with PSA.

References: None

Disclosure of Interests: None declared

DOI: 10.1136/annrheumdis-2020-eular.5495

\section{AB0765 \\ CAN A 6-JOINT ULTRASOUND SCORE DIFFERENTIATE RHEUMATOID ARTHRITIS FROM PSORIATIC ARTHRITIS? A CROSS SECTIONAL STUDY}

F. Figus $^{1}$, L. Idolazzi ${ }^{2}$, P. Perić ${ }^{3}$, A. Zabotti ${ }^{4}$, I. Tinazzi ${ }^{2}$, I. Azzolin ${ }^{1}$,

E. Montabone5, T. Sapundzhieva ${ }^{6}$, A. Batalov ${ }^{7}$, P. Todorov ${ }^{7}$, R. Karalilova ${ }^{6}$ A. Iagnocco ${ }^{1} .{ }^{1}$ Universita Di Torino, Torino, Italy; ${ }^{2}$ Universita' Di Verona, Verona, Italy; ${ }^{3}$ Universita' Di Zagreb, Zagreb, Croatia; ${ }^{4}$ Universita' Di Udine, Udine, Italy; ${ }^{1}$ Universita Di Torino, Torino, Italy; ${ }^{6}$ Universita' Di Plovdiv, Plovdiv, Bulgaria; ${ }^{7}$ Universit' Di Plovdiv, Plovdiv, Bulgaria

Background: Ultrasonography (US) is a well established technique both for diagnosis and follow up in Rheumatoid Arthritis (RA) and Psoriatic Arthritis (PSA) ${ }^{1}$. To date, there is no consensus regarding a standardized US evaluation of joint involvement by a validated score to be used for differential diagnosis. Objectives: 1) To differentiate the ultrasound features of patients affected by active RA or active PSA by using a 6 -joints score ${ }^{2}$. 2) To analyze correlations between those findings and clinical patterns of PSA disease.

Methods: 68 RA and 38 PSA patients (divided in two equal subgroups according to the clinical involvement i.e. polyarticular or oligoarticular) were enrolled in a multi-center cross-sectional study. All patients underwent clinical evaluation including demographic data, disease characteristics, laboratory test and tender/ swollen joints count. SDAI and DAPSA were calculated in accordance to the disease and standard of care. The sonographic evaluation of wrists, II MCFs and knees, was performed using a multifrequency linear probe $(13-18 \mathrm{MHz})$ with power Doppler $(7.5 \mathrm{MHz}$, PRF500 Hz). High-end equipment was used and the scanning technique as well as the lesion assessment was previously agreed among the participants, who performed a consensus session 100 images. A validated ultrasound score $^{2}$, which included effusion, synovial hypertrophy and synovial hypervascularization at 6 joints sites was used. Those lesions were assessed according to OMERACT definitions and semi-quantitatively graded (0-3). By summing the scores obtained at each joint site and globally, a joint score for articular involvement, a score for the severity of each lesion and a global 6-joint score for all abnormalities were calculated.

Results: Clinical evaluation showed no statistically significant differences between RA and PSA (table I). Ultrasound detected significant differences in the score of joint effusion (SE) $(p<0.021)$, synovial hypertrophy $(\mathrm{SH})(\mathrm{p}<0.001)$ and Doppler signal $(p<0.011)$ between oligoarticular PSA and RA. Significant differences in the joint score of II MCF $(p<0.000)$ and wrist $(p<0.032)$ were also found between oligoarticular PSA and RA. The global 6-joint score was 10,88 in RA 6,05 in PSA oligoarticular, 16,32 in PSA polyarticular. No differences were found between RA and polyarticular PSA.

Conclusion: Ultrasound evaluation of 6 target joints might help to discriminate RA and PSA oligoarticular subset. The study of a limited number of joints is 